\title{
MINIATURE THICK FILM RESISTORS FOR MICROWAVE INTEGRATED CIRCUITS
}

\author{
ZBIGNIEW SZCZEPAŃSKI \\ Warsaw University of Technology, Institute of Microelectronics and Optoelectronics \\ (Received May 29, 1990; in final form August 23, 1990)
}

In this paper the results of miniature thick film resistors designed as add-on lumped resistors are presented. A few resistor configurations have been elaborated and tested, and a specific trimming resistance, which avoids parasitic trimming inductance was used. Some of the designed resistors were used in isolators and directional couplers for the $2-11 \mathrm{GHz}$ band. The electrical parameters have been presented and discussed. The assembly methods for these resistors to the microstrip lines have also been given.

\section{INTRODUCTION}

Microwave integrated circuits (MIC) technology has progressed rapidly in the last years. At the same time, many new kinds of active and passive components for the MIC's have been developed. Presently most widely used construction of the microwave integrated circuits are hybrid microwave integrated circuits, based on microstrip lines, which play the role of a wave guide. In these circuits, except for the distributed elements, the lumped-elements such as resistors, capacitors and diodes often exist.

The resistance MIC's elements can be used as isolators load, in attenuators, as matching elements, and as shunt to ground $50 \Omega$ terminations. The resistors for MIC's should guarantee a low temperature coefficient of resistance (TCR), good resistance stability, small dimensions, easy attachment to the microstrip line, and ample power handling ${ }^{2}$.

Low TCR and good stability involve small time and temperature resistance changes. The resistor sizes for microwave application should be very small. When they are much smaller than the applicable wavelength, the resistors behave like lumped-element resistors, at which the parasitic effects are also minimized. As a rule the largest dimension of the resistor should be less than $0.1 \lambda$ at the highest frequency.

But one should note that too small dimensions may cause some processing and manufacturing problems. Larger dimensions allow greater power dissipation but the maximum resistor dimension is limited by the increasing parasitic. Another problem concerning a strip line dimension is the continuity of transmission line where the resistor is connected. It would be desirable that the resistor width approximated the strip line width. 
Since the limiting frequency of a skin effect for a thick resistive film is very large and an electromagnetic field penetrates the whole resistive film thickness, the resistance value can be described as:

$\mathrm{R}=\mathrm{R}_{\square} \frac{L}{W}=Z_{o}$

There: $\mathrm{R}_{\square}$ is the sheet resistivity, and $L$ and $W$ are the film length and width, respectively. The resistance value can thus be defined by counting the number of squares between the conductors pads. When a resistor is attached to a strip line as the matching element, its resistance should be matched to the input strip line impedance $Z_{o}$. Thus these resistors must be made with a good resistance tolerance.

\section{EXPERIMENTAL}

The choice of the configuration and resistor technology for the microwave resistors was determined by such factors as the need for miniaturization of such resistors, low production cost, good resistance stability, high power carrying capacity of resistive film, and good resistance for environmental conditions ${ }^{1}$.

Taking into account different applications of these resistors and different operating frequencies, a few resistor configurations have been designed:

-Plane configuration

- Spatial configuration

- Mixed configuration.

These configurations differ from the distribution of conductor pads as well as from different dimensions.

For the realization of these resistors, thick film technology has been chosen, since thick resistive films based on ruthenium have been characterized by high power handling capability (up to $15 \mathrm{~W} / \mathrm{cm}^{2}$ ). It allows the dissipation of considerable power on a small surface of resistive film.

All the manufacturing processes of thick film resistors have been based on Polishmade materials, as well as substrates and conductive and resistive compositions. The realized resistors have been tested in order to define electrical and RF performances.

All resistors were examined for long term stability, TCR, VSWR, and power handling capability. Before measurements of the resistors, they were aged at $150^{\circ} \mathrm{C}$ for 36 hours to stabilize their properties; failures were eliminated at this stage.

The main advantage of designed resistors is the specific applied trimming $\operatorname{method}^{4}$. Resistors are often trimmed by laser trimming or air abrasive trimming techniques. The laser trimming increases inductance at microwave frequencies since the microwave signal path becomes longer. (Fig. 1). On the other hand, laser trimming reduces power handling capability. Therefore laser trimming cannot be used for resistors to be operated at high power dissipation and high frequencies ${ }^{2}$. 


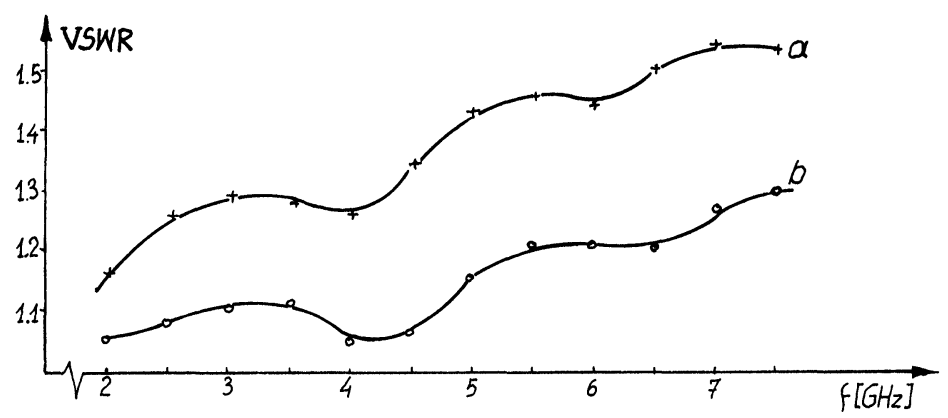

FIGURE 1 The effect of laser cut for RF performance resistor: a-resistor with laser cut; $b$-resistor without cut.

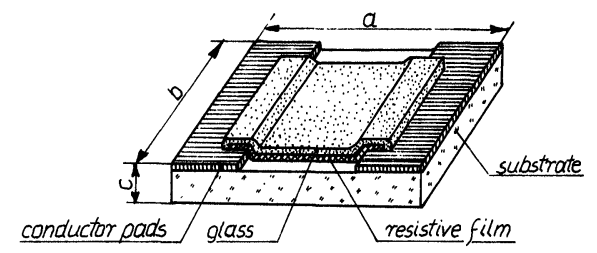

FIGURE 2 The scheme of plane resistor configuration.

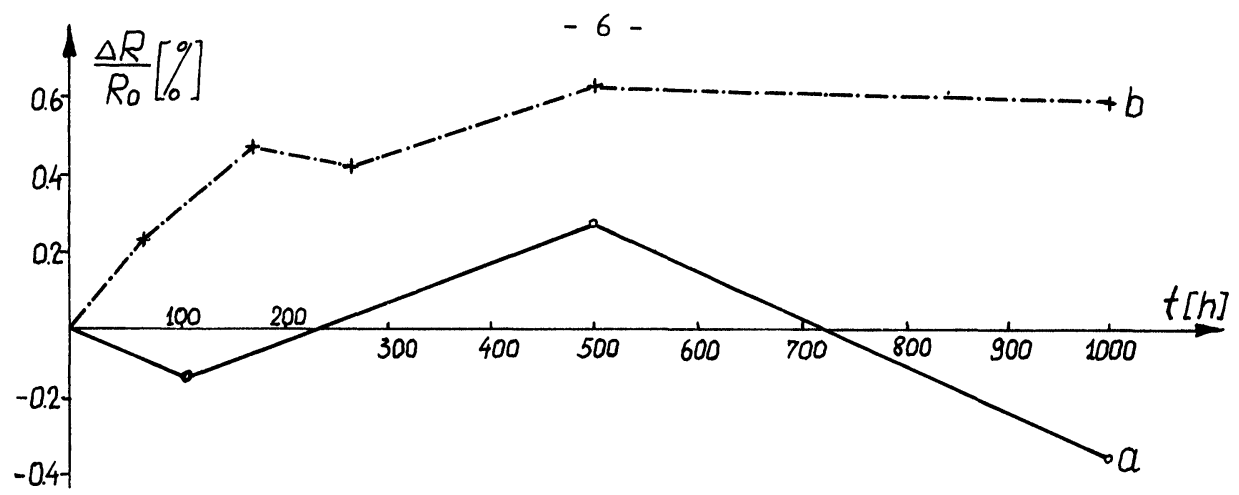

FIGURE 3 Long-term resistance stability for plane resistors: a-at 398K without load; b-at 343K with electric load.

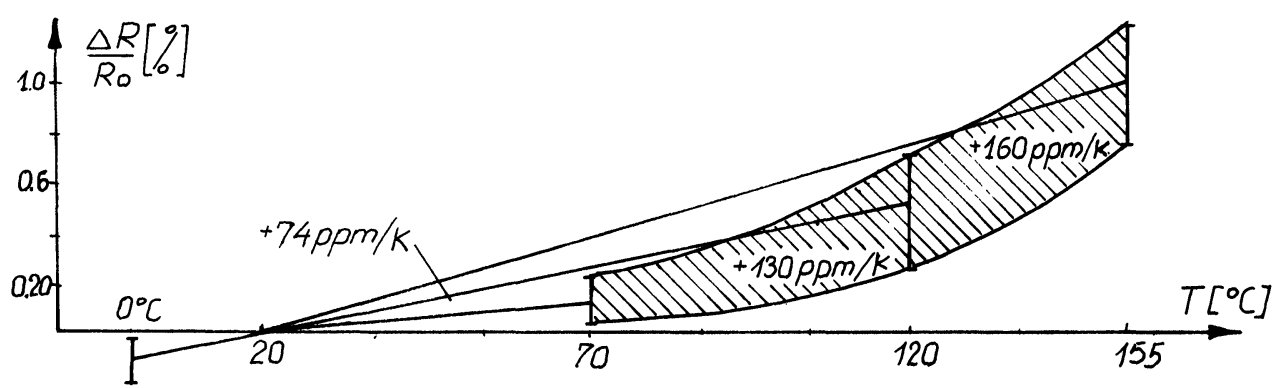

FIGURE 4 Resistance changes as a function of temperature. 
Trimming by means of changes in the resistive film thickness cannot be used either, since it decreases the resistance stability.

To achieve a good tolerance of resistance, the designed and fabricated resistors were trimmed by thermal trimming through one, two or several stages. This method permits a resistance tolerance to 0.5 per cent. It must be noted that with thermal trimming, the resistors after firing should have tolerance of not more than 10-15 per cent. In this case thick film processes will require more careful preparation of both conductor and resistor artwork.

In all the designed and fabricated resistors, the resistive film was coated with a glass composition, which affords resistors a protection from environmental conditions.

In Fig. 1 the effect of laser cut for VSWR can be seen.

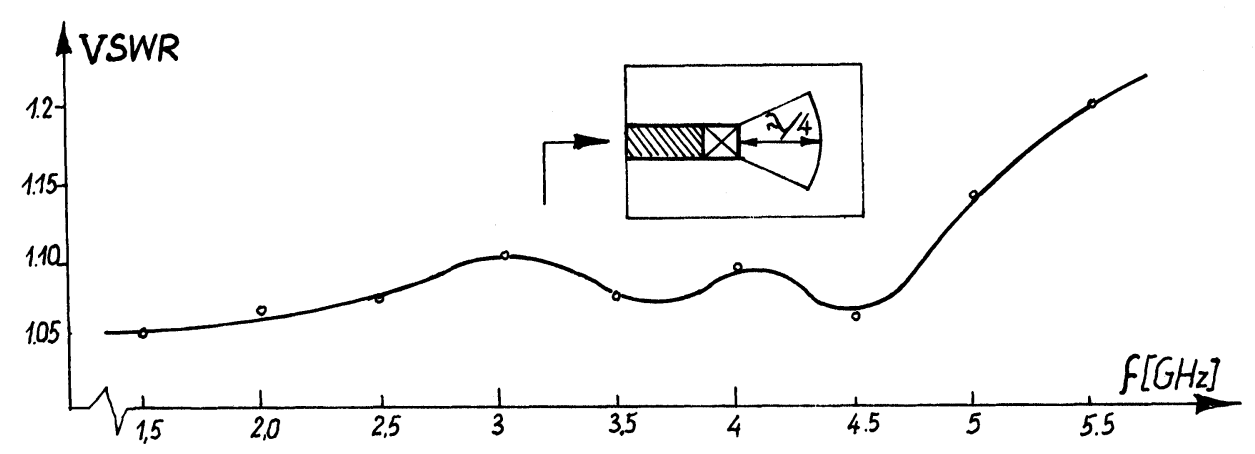

FIGURE 5 VSWR vs. frequency for plane resistors.

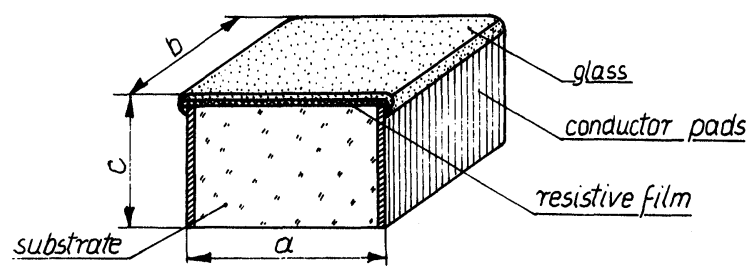

FIGURE 6 Spatial resistor configuration.

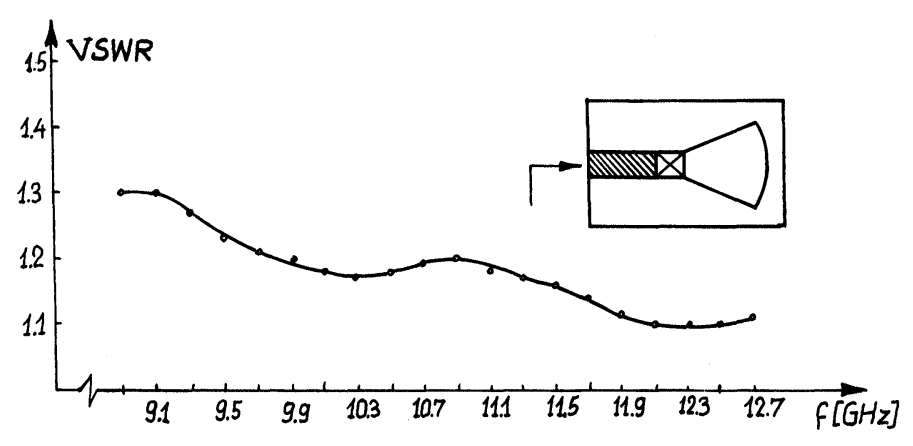

FIGURE 7 VSWR vs. frequency for spatial resistors. 


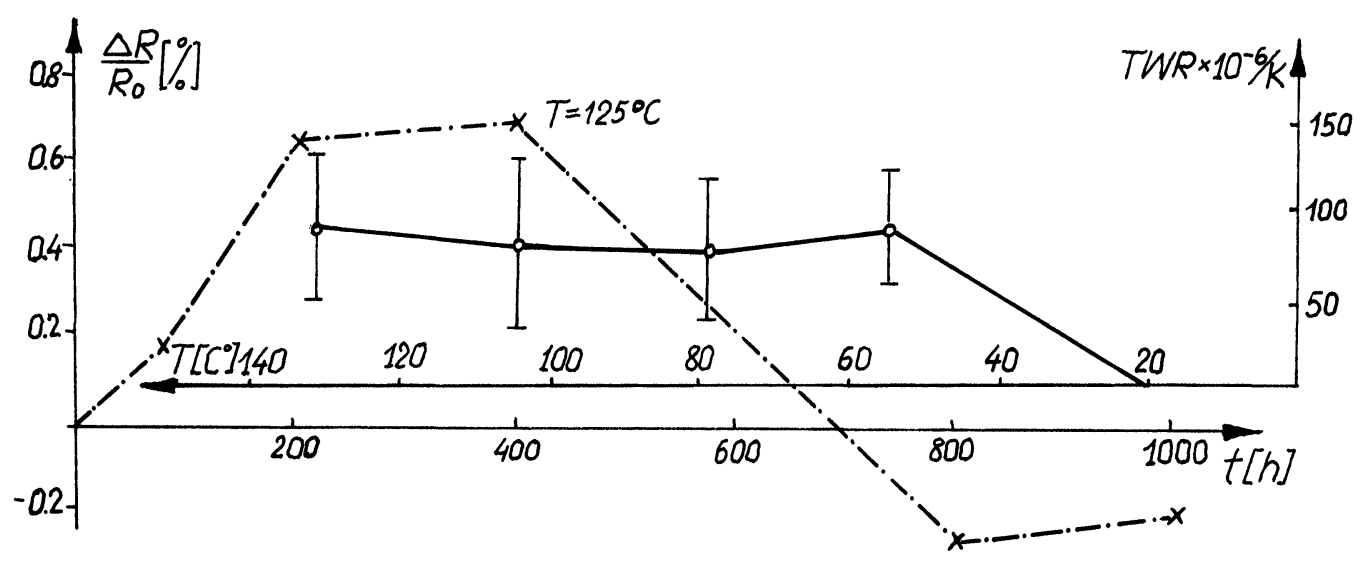

FIGURE 8 Long-term resistance stability and the changes of TCR with temperature for spatial resistors.

\section{EXPERIMENTAL RESULTS}

The first configuration (Fig. 2) is characterized by the resistive and conductive layers that are located on the same surface. Such a shape avoids parasitic capacitance connected with resistor. Attachment of the resistor to the microstrip line is achieved by soldering using the "flip chip" technique. Plane resistors are characterized by a good long term resistance stability as shown in Fig. 3.

The changes in termperature coefficient of resistance with temperature for the plane resistors are shown in Fig. 4.

To define the resistors properties at microwave frequencies the resistors were attached to $50 \mathrm{ohm}$ transmission line with a $1 / 4 \lambda$ open circuit stub (Fig. 5). The second configuration results in a high degree of miniaturization as resistive and conductive layers are spaced on three separate planes of a cubic body (Fig. 6). The smallest sizes obtained for such resistors were $0.7 \times 0.7 \times 0.5 \mathrm{~mm}$. Such resistors are recommended for higher band frequency since they are characterized by low VSWR over the operating frequency (Fig. 7). Thermal measurements for spatial resistors are shown in Fig. 8. The measured resistive film temperature was below $398 \mathrm{~K}$ during the thermal resistance stability with an electric loading of $140 \mathrm{~mW}$ on

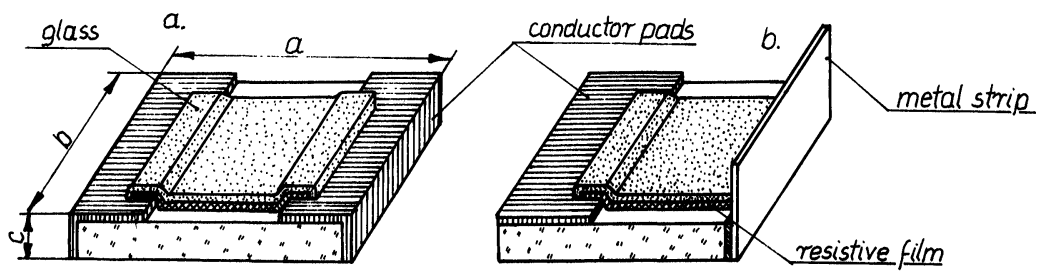

FIGURE 9 Mixed resistor configuration: a-the configuration with dual conductor pads; b-asymmetric resistor configuration. 
every resistor. Because of the small sizes of these resistors, problems with the precision position and placement arise with their attachment.

In the last configuration, i.e., mixed configuration, the conductive film is put on two or three planes of the body (Fig. 9). The resistors with double conductive pads are relatively easy to assemble. The configuration " $8 \mathrm{~b}$ " with one metal strip is especially recommended when it is used as a shunt to ground (Fig. 10). The electric and microwave properties of the resistors with double conductor pads of $1.8 \times 0.8$ $\times 0.5 \mathrm{~mm}$ are given in Fig. 11 and Fig. 12. The general parameters for the fabricated resistors have been compiled in Table 1 .

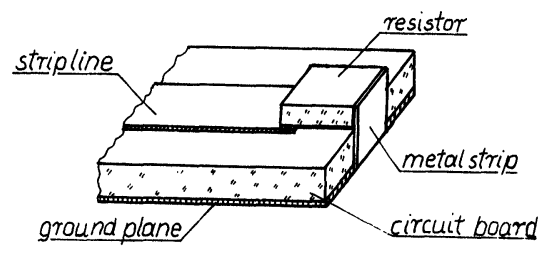

FIGURE 10 The scheme of RF grounding achieved with metal strip.

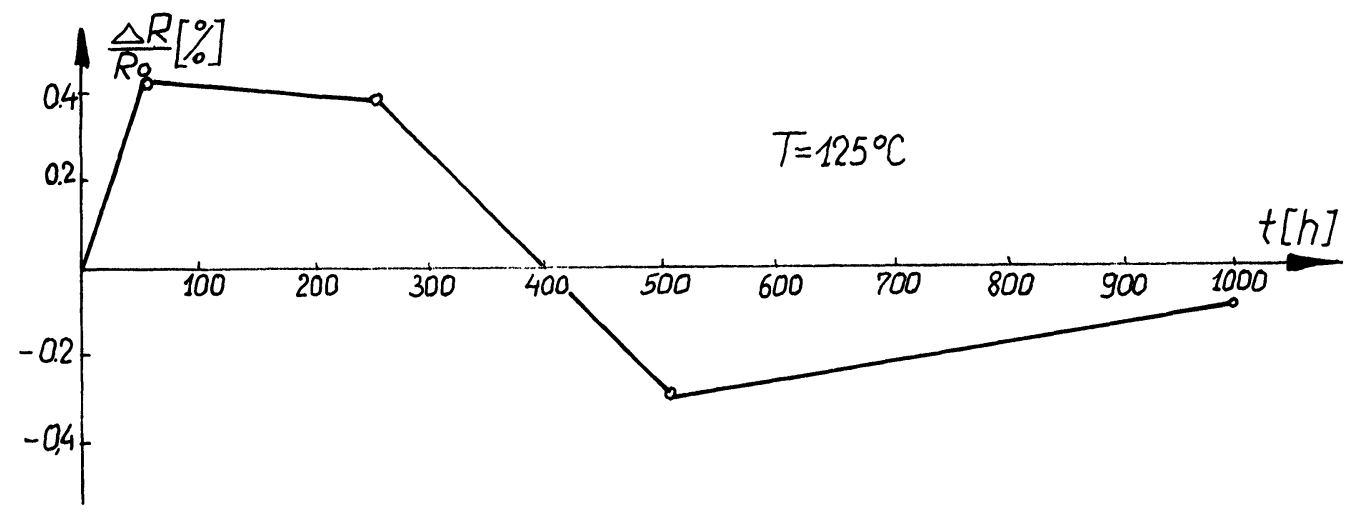

FIGURE 11 Long-term stability for mixed resistor configuration.

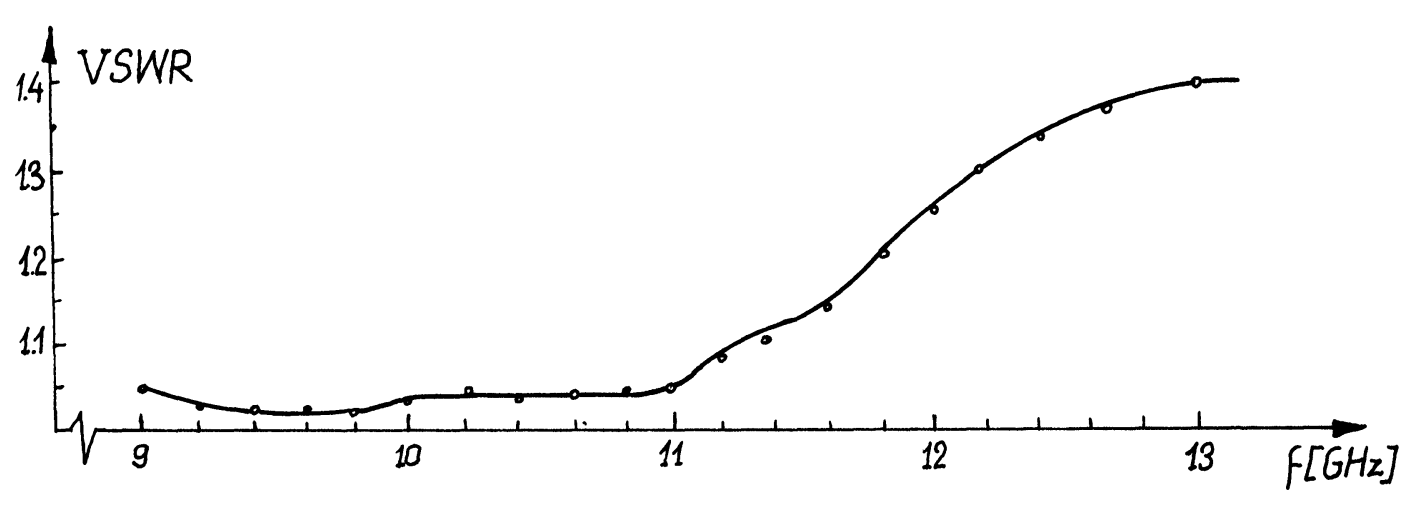

FIGURE 12 VSWR vs. frequency for mixed resistor configuration. 


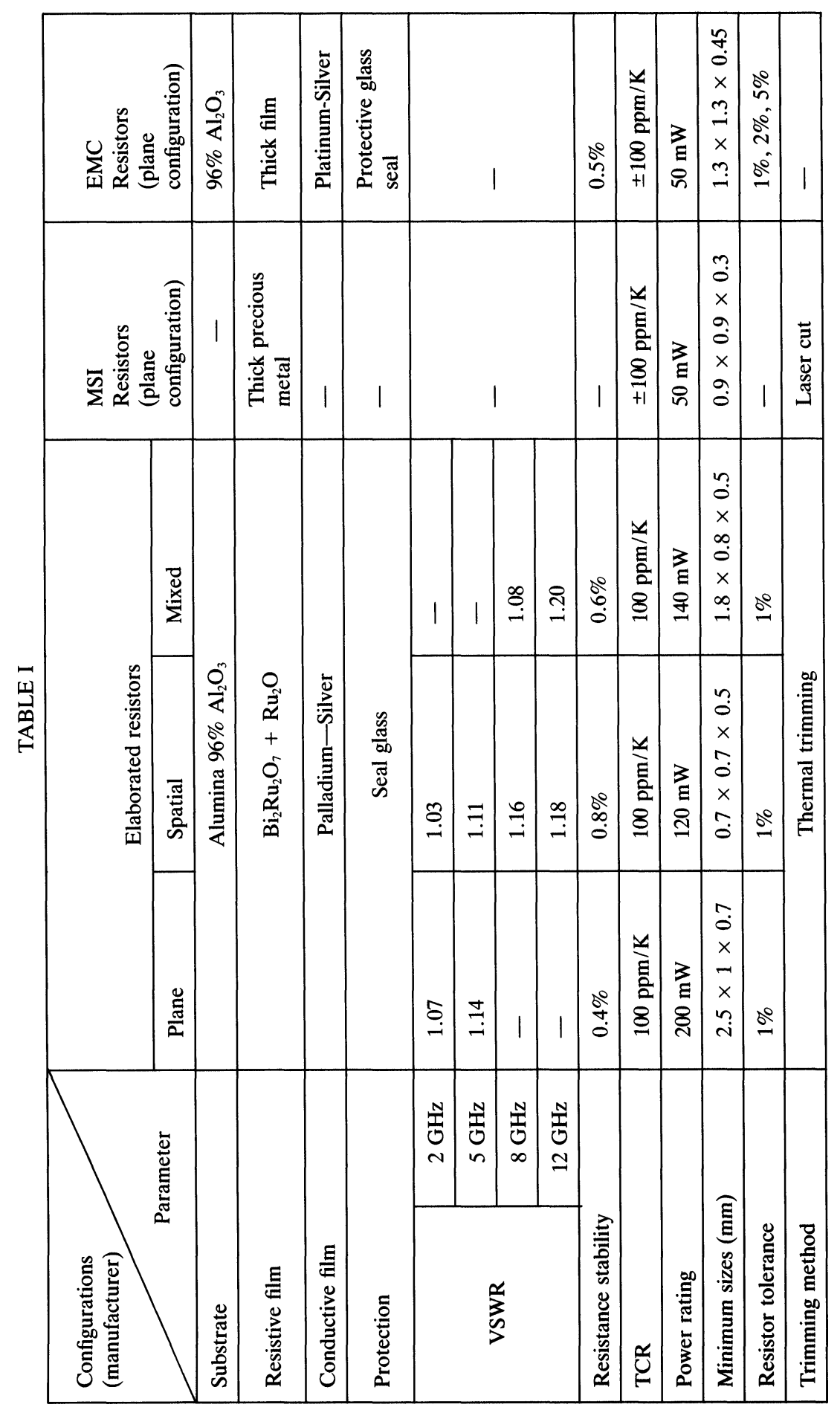




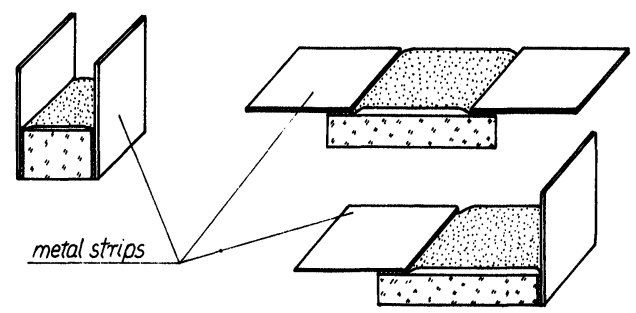

FIGURE 13 The scheme of chip resistors with metal strips.

\section{MOUNTING RECOMMENDATIONS FOR CHIP RESISTORS}

Designed and elaborated resistors are available as leadless chips or chips with metal strips (Fig. 13). These metal strips make chip resistor attachment easy. They are made of copper and are electroplated with gold. The metal strips are attached to the thick film metalization with the use of microgap welding or eutectic solder with high melting temperature (i.e. $\mathrm{Sn} 96 \mathrm{Ag} 4$, te $=220^{\circ} \mathrm{C}$ ). When choosing an attachment technique, great care must be given to achieving the desired RF performance.

In all these cases $50 \mathrm{ohm}$ microstrip lines are the most widely used for these components. Any device parasitic reactances can usually be compensated by external circuitry. In general, it is essential to minimize the capacitance to ground for attenuation of 1 to $8 \mathrm{~dB}$ values. This can be accomplished by a large low dielectric ground plane spacing. In all cases, grounding is critical. For terminating resistors the same rules apply to the ground connections. The parasitics associated with termination connections for in-line resistors should be minimized. This can be realized by matching to the stripline width, both the chip pad sizes and the attachment method. Epoxy bonding and various soldering methods are mainly used for attachment of chip resistors.

Epoxy bonding is suitable for most applications. For the mounting of chip resistors, silver or gold conductive epoxies are used; silver epoxy is commonly used due to its low cost and good bonding with palladium silver and platinum silver terminations.

The epoxy can be applied by screening or dispensing on the substrate surface to both leadless chip resistors and chips with metal strips. Chip resistor soldering can be made with reflow soldering in which solder preforms, solder paste or pre-

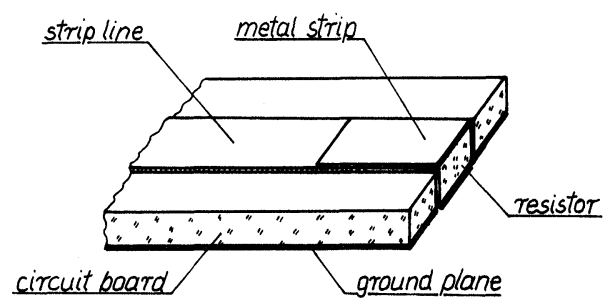

FIGURE 14 The scheme of the attachment of spatial chip resistor with metal strips. 
tinning can be used. For chip resistors with a plane configuration, the flip-chip technique with pretinning of resistor pads is most often suitable.

The miniature chip resistors with a spatial configuration are attached to the strip line by the means of epoxy bonding or soldering with an iron. In this case, prior to making epoxy or solder electrical connections to the chip, it must be held in place using non-conductive epoxy, ensuring that the chip does not move when soldering.

The spatial chip configuration with metal strips is the most suitable when the resistor is located in the cavity of a substrate (Fig. 14).

The chip resistors with metal strips can be soldered to circuit board using solder with paste or in wire form or melted by an iron, torch, or other localized source.

\section{CONCLUSIONS}

From the above experimental data, the following conclusions can be drawn:

-Designed thick film chip resistors can be used in microwave integrated circuits in the operating frequency up to $\mathrm{X}$ band

- These resistors are characterized by good resistance stability and satisfactory values of TCR

- Chip resistors offer good RF performances up to $12 \mathrm{GHz}$ with VSWR better than 1.22

- They can be easily attached to microstrip lines by the means of conventional assembly methods

-Plane and spatial chip resistors have been used in isolators for L-S and X band and the achieved parameters were satisfactory.

\section{REFERENCES}

1. Larry Boss; State of the Art in Hybrid Circuits Resistors. Hybrid Circuits Technology. vol. 6, No. 10, October 1989.

2. Chen Y. Ho; VSWR, Power Dissipation: Keys to Film Resistors Microwaves. December 1981, pp. 69-78.

3. G. Stecher, R. Bosch; Thick Film Resistors not only on Ceramics: How to Obtain Suitable Layout Parameters. Hybrid Circuits No. 17, September 1988.

4. Z. Szczepański, R. Kisiel, J. Kalenik; Thick Film Mini Chip Resistors for Microwave Applications. 32. Internationales Wissenschaftliches Kolloquium. Ilmenau/DDR/, October 1988. 

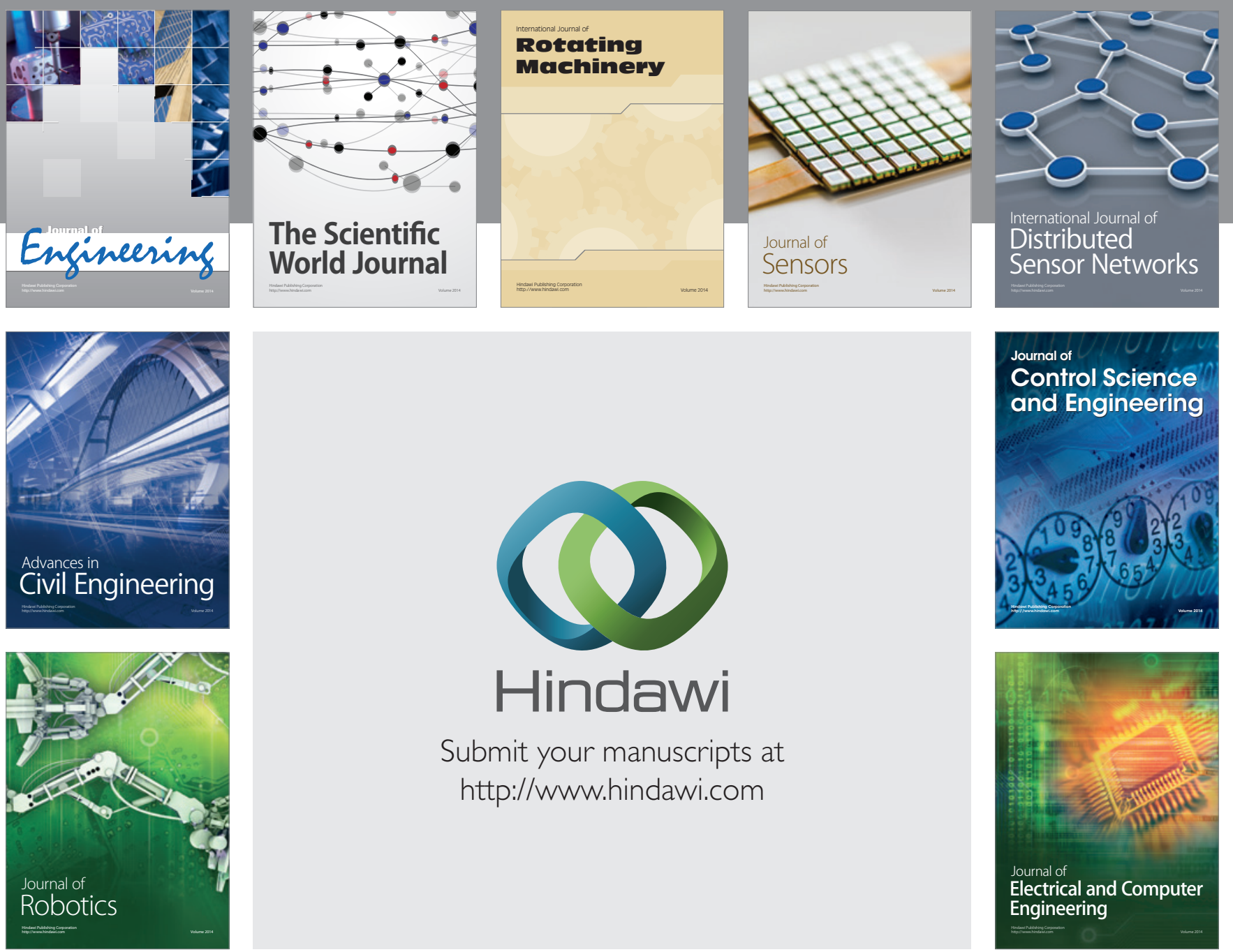

Submit your manuscripts at

http://www.hindawi.com
